\title{
ON THE RIEMANN-HARDY CONJECTURE FOR THE RAMANUJAN ZETA-FUNCTION
}

\author{
XIAO-JUN YANG
}

\begin{abstract}
In this article we propose the integral, series and product representations for the Ramanujan zeta-function. We suggest a variant for the Conrey-Ghosh product for the entire Ramanujan zeta-function. We present some variants for the product for the Ramanujan $\Xi$-function. We prove that all of its zeros are real. Along the way we obtain the truth of the Riemann-Hardy conjecture.
\end{abstract}

\section{Contents}

1. Introduction 1

2. The integral and series representations 4

3. The product formulas 10

4. Real zeros 12

5. The nontrivial zeros 15

6. The equivalent theorems 16

$\begin{array}{ll}\text { References } & 24\end{array}$

\section{INTRODUCTION}

In his remarkable paper [1], Ramanujan in 1916 introduced the zeta-function $L_{\tau}(s)$, that in his honor is now called the Ramanujan zeta-function, which is defined as [2]

$$
L_{\tau}(s)=\sum_{k=1}^{\infty} \frac{\tau(k)}{k^{s}}(R e(s)>13 / 2)
$$

1991 Mathematics Subject Classification. Primary:11-04; Secondary: 11Y35; $11 Y 55$.

Key words and phrases. Ramanujan zeta-function, Ramanujan's tau-function, entire Ramanujan zeta-function, Ramanujan $\Xi$-function, Riemann-Hardy conjecture. 
and conjectured the arithmetical function $\tau(n)$ that in his honor is now called the Ramanujan's tau-function, which is expressed by (for example, see [3], p.156)

$$
G(x)=\sum_{n=0}^{\infty} \tau(n) x^{n}=x \prod_{n=1}^{\infty}\left(1-x^{n}\right)^{24}(|x|<1),
$$

which was proved by Mordell in $1917[4,5]$, where $s \in \mathbb{C}, x \in \mathbb{R}$ and $k \in \mathbb{N}$, if we denote the sets of the complex numbers, natural numbers and real numbers by $\mathbb{C}, \mathbb{N}$ and $\mathbb{C}$, and the real and imaginary parts of $s \in \mathbb{C}$ by $\operatorname{Re}(s)$ and $\operatorname{Im}(s)$, respectively. Before the work of Deligne [6], Ramanujan conjectured an Euler product for Eq. (1), which can be expressed as $[2,7,8]$

$$
L_{\tau}(s)=\prod_{p}\left(1-\tau(n) p^{-s}+p^{11-2 s}\right)^{-1}=\prod_{p}^{\infty}\left(1-\alpha_{1} p^{-s}\right)^{-1}\left(1-\overline{\alpha_{2}} p^{-s}\right)^{-1}
$$

where $p$ is prime and $\operatorname{Re}(s)>13 / 2$. The second term of Eq. (3) (see [3], p.164) was conjectured by Ramanujan [1] and proved by Mordell in 1917 [4], and the third term of Eq. (3) was proved by Deligne [6]. Rankin in 1939 [9] suggested the entire Ramanujan zeta-function $\xi_{\tau}(s)$ by

$$
\xi_{\tau}(s)=(2 \pi)^{-s} \Gamma(s) L_{\tau}(s)
$$

which leads to the functional equation

$$
\xi_{\tau}(s)=\xi_{\tau}(12-s),
$$

proved by Wilton [10] and denoted by Conrey and Ghosh [2]. By the result of the Weierstrass and Hadamard work on the entire functions [11], Conrey and Ghosh pointed out that the entire Ramanujan zeta-function of order $\alpha=1$ can be rewritten in the form [2]

$$
\xi_{\tau}(s)=\xi_{\tau}(0) e^{\gamma s} \prod_{\vartheta=1}^{\infty}\left(1-\frac{s}{\rho_{\vartheta}}\right) e^{s / \rho_{\vartheta}}
$$

where $\rho_{\vartheta}$ run the nontrivial zeros of $\xi_{\tau}(s), \vartheta \in \mathbb{N}$ and $\gamma$ is a constant. For $\varpi \neq 0$ and $i=\sqrt{-1}$, Rankin in 1939 showed [9]

$$
\Delta_{\tau}(\varpi)=\frac{1}{\varpi^{12}} \Delta_{\tau}\left(-\frac{1}{\varpi}\right)
$$

which implies that [12]

$$
\xi_{\tau}(s)=\int_{0}^{\infty} \varpi^{s-1} \Delta_{\tau}(i \varpi) d \varpi
$$


RIEMANN-HARDY CONJECTURE

and (see $[11,12]$, which is the special case of Wilton [10])

$$
\Xi_{\tau}(t)=\xi_{\tau}(6+i t)=\int_{0}^{\infty} \varpi^{5+i t} \Delta_{\tau}(i \varpi) d \varpi=\int_{-\infty}^{\infty} F(l) e^{i l t} d l
$$

where

$$
F(l)=\Delta_{\tau}\left(i e^{l}\right) e^{6 l}
$$

and [13]

$$
\Delta_{\tau}(\varpi)=\eta(\varpi)^{24}=e^{\varpi} \prod_{k=1}^{\infty}\left(1-e^{k \varpi}\right)^{24}=\sum_{k=1}^{\infty} \tau(k) e^{2 \pi i k \varpi}
$$

with

$$
\eta\left(-\frac{1}{\varpi}\right)=\sqrt{\frac{\varpi}{i}} \eta(\varpi),
$$

which satisfies the Dedekind eta function [14]

$$
\eta(\varpi)=e^{\varpi / 24} \prod_{k=1}^{\infty}\left(1-e^{k \varpi}\right) .
$$

Following the idea of Chirre and Castañón [15], $\Xi_{\tau}(t)$ is called the Ramanujan $\Xi$ function. Based on the above results mentioned, the interesting topics for the Ramanujan zeta-function have been conjectured by Hardy as follows (see [3], p.174):

- (Critical Strip) The critical strip for the Ramanujan zeta-function is $11 / 2<R e(s)<13 / 2$;

- (Trivial Zeros) The trivial zeros for the Ramanujan zeta-function is $s=-w$ with $w \in \mathbb{N} \cup\{0\}$;

- (Riemann-Hardy Conjecture)

Conjecture 1. The nontrivial zeros for the Ramanujan zeta-function lie on the critical line $R e(s)=6$.

Hardy in 1940 (see [3], p.174) proposed the Riemann-Hardy conjecture for the Ramanujan zeta-function associated with the Ramanujan's tau-function, that in his honor is as an analogue of Riemann conjecture for the Riemann zeta-function. Up to now, it remains an important unsolved problem in the analytic number theory. Moreover, a great many of the interesting problems similar to the interesting topics for the Riemann zeta-function have been reported by Hardy [3]. For example, an analogue of the theorem of Hardy for the entire Ramanujan zeta-function was proposed by Hardy (see [3], p.174) and proved by Lekkerkerker [16]. An analogue of 
the von Mangoldt-like formula for the entire Ramanujan zeta-function was conjectured by Hardy (see [3], p.174) and proved by Ki [17]. Ferguson and coauthors [18] reported 18 nontrivial zeros for the Ramanujan zeta-function. An analogue of the Rieman-Siegel-like formula for the Ramanujan zeta-function (1) was conjectured by Hardy (see [3], p.174) and proved by Keiper [19].

Let $\varphi_{\vartheta}$ run the positive zeros for $\Xi_{\tau}(t)$. In 1983, Hafner [13] proposed the equivalent form of Conjecture 1, which states that

$$
N_{0}(T)=N(T)
$$

where

$$
N_{0}(T)=\#\left\{s=6+i t: 0 \leq t \leq T, \Xi_{\tau}(s)=0\right\}
$$

and

$$
N(T)=\#\left\{s=\sigma+i t: 0 \leq t \leq T, 11 / 2<\sigma<13 / 2, \xi_{\tau}(s)=0\right\}
$$

Moreover, other statements equivalent to Conjecture 1 were reported in Moreno [7]. The main targets of our article are to proceed to prove Conjecture 1, and to propose the series and product formulas to give the structure of the product formulas to obtain this conjecture. The structure of this article is given as follows. In Section 2 we propose the integral and series representations for (1) and the Lekkerkerker theorem. In Section 3 we suggest the product formulas for (1) and (4). In Section 4 we prove that all zeros of the Ramanujan $\Xi$-function (9) are real. In Section 5 we also prove the truth of Conjecture 1. Finally, we propose some equivalent theorems in Section 6.

\section{The IntEgral AND SERIES REPRESENTATIONS}

In this section we consider the integral and series representations for the Ramanujan zeta-function.

Now we consider the remark on the work of Wilton to consider the integral representations of them.

Remark. In 1929, Wilton (see [10], formula (5.2)) proved that

$$
\xi_{\tau}(s)=\int_{1}^{\infty}\left(\varpi^{s-6}+\varpi^{6-s}\right) \varpi^{6} G\left(e^{-2 \pi \varpi}\right) \frac{d \varpi}{\varpi},
$$


which leads to

$$
\begin{aligned}
& \xi_{\tau}(s) \\
& =\int_{1}^{\infty}\left(\varpi^{s-6}+\varpi^{6-s}\right) \varpi^{6} G\left(e^{-2 \pi \varpi}\right) \frac{d \varpi}{\varpi} \\
& =2 \int_{1}^{\infty}\left(\frac{e^{(s-6) \log \varpi}+e^{-(s-6)}}{2}\right) \varpi^{6} G\left(e^{-2 \pi \varpi}\right) \frac{d \varpi}{\varpi} \\
& =2 \int_{1}^{\infty} \cosh ((s-6) \log \varpi) \varpi^{5} G\left(e^{-2 \pi \varpi}\right) d \varpi .
\end{aligned}
$$

Thus, we have

$$
\xi_{\tau}(s)=2 \int_{1}^{\infty} \cosh ((s-6) \log \varpi) \mathbb{X}(\varpi) d \varpi
$$

where

$$
\mathbb{X}(\varpi)=\varpi^{5} G\left(e^{-2 \pi \varpi}\right)
$$

Theorem 1. Let $\Delta_{\tau}(\varpi)=\sum_{k=1}^{\infty} \tau(k) e^{k \varpi}$. If $\xi_{\tau}(s)$ is defined in (4), then we have for $s \in \mathbb{C}$,

$$
\xi_{\tau}(s)=2 \int_{1}^{\infty} \cosh ((s-6) \log \varpi) \mathbb{X}(\varpi) d \varpi
$$

where

$$
\mathbb{X}(\varpi)=\varpi^{5} G\left(e^{-2 \pi \varpi}\right)=\varpi^{5} \Delta_{\tau}(i \varpi)
$$

Proof. By the definition of the entire Ramanujan zeta-function and (7), we have

$$
\begin{aligned}
& \xi_{\tau}(s) \\
& =\int_{0}^{\infty} \varpi^{s-1} \Delta_{\tau}(i \varpi) d \varpi \\
& =\int_{1}^{\infty} \varpi^{s} \Delta_{\tau}(i \varpi) \frac{d \varpi}{\varpi}+\int_{0}^{1} \varpi^{s} \Delta_{\tau}(i \varpi) \frac{d \varpi}{\varpi} \\
& =\int_{1}^{\infty} \varpi^{s} \Delta_{\tau}(i \varpi) \frac{d \varpi}{\varpi}-\int_{1}^{\infty} \varpi^{12-s} \Delta_{\tau}(i \varpi) \frac{d \varpi}{\varpi} \\
& =\int_{1}^{\infty}\left(\varpi^{s}-\varpi^{12-s}\right) \Delta_{\tau}(i \varpi) \frac{d \varpi}{\varpi} \\
& =\int_{1}^{\infty}\left(\varpi^{s-6}-\varpi^{6-s}\right) \varpi^{6} \Delta_{\tau}(i \varpi) \frac{d \varpi}{\varpi}
\end{aligned}
$$


and

$$
\int_{0}^{1} \varpi^{s} \Delta_{\tau}(i \varpi) \frac{d \varpi}{\varpi}=-\int_{1}^{\infty} \varpi^{12-s} \Delta_{\tau}(i \varpi) \frac{d \varpi}{\varpi}
$$

where

$$
\Delta_{\tau}(i \varpi)=\frac{\Delta_{\tau}(-1 / i \varpi)}{\varpi^{12}} .
$$

By (2) and (11), we arrive at the identity

$$
G\left(e^{-2 \pi \varpi}\right)=\Delta_{\tau}(i \varpi),
$$

which leads to

$$
\mathbb{X}(\varpi)=\varpi^{5} G\left(e^{-2 \pi \varpi}\right)=\varpi^{5} \Delta_{\tau}(i \varpi)
$$

It follows that

$$
\begin{aligned}
& \xi_{\tau}(s) \\
& =\int_{1}^{\infty}\left(\varpi^{s-6}-\varpi^{6-s}\right) \varpi^{6} \Delta_{\tau}(i \varpi) \frac{d \varpi}{\varpi} \\
& =\int_{1}^{\infty}\left(e^{(s-6) \log \varpi}-e^{-(s-6)}\right) \varpi^{5} \Delta_{\tau}(i \varpi) d \varpi \\
& =2 \int_{1}^{\infty}\left(\frac{e^{(s-6) \log \varpi-e^{-(s-6)}}}{2}\right) \varpi^{5} \Delta_{\tau}(i \varpi) d \varpi \\
& =2 \int_{1}^{\infty} \cosh ((s-6) \log \varpi) \varpi^{5} \Delta_{\tau}(i \varpi) d \varpi \\
& =2 \int_{1}^{\infty} \cosh ((s-6) \log \varpi) \mathbb{X}(\varpi) d \varpi .
\end{aligned}
$$

Then, the desired result follows.

Hence, we finish the proof of Theorem 1.

Theorem 2. Let $\Delta_{\tau}(\varpi)=\sum_{k=1}^{\infty} \tau(k) e^{k \varpi}$. If $\xi_{\tau}(s)$ is defined in (4), then we have for $m \in \mathbb{N} \cup\{0\}$ and $s \in \mathbb{C}$,

$$
\xi_{\tau}(s)=\sum_{m=0}^{\infty} \beta_{m}(s-6)^{2 m}
$$

where

$$
\beta_{m}=\frac{2}{\Gamma(2 m+1)} \int_{1}^{\infty}(\log \varpi)^{2 m} \mathbb{X}(\varpi) d \varpi
$$


Proof. By Theorem 1, we now show that

$$
\begin{aligned}
& \xi_{\tau}(s) \\
& =2 \int_{1}^{\infty} \cosh ((s-6) \log \varpi) \mathbb{X}(\varpi) d \varpi \\
& =2 \int_{1}^{\infty} \sum_{m=0}^{\infty} \frac{((s-6) \log \varpi)^{2 m}}{\Gamma(2 m+1)} \mathbb{X}(\varpi) d \varpi \\
& =\sum_{m=0}^{\infty}\left(2 \int_{1}^{\infty} \frac{(\log \varpi)^{2 m}}{\Gamma(2 m+1)} \mathbb{X}(\varpi) d \varpi\right)(s-6)^{2 m} \\
& =\sum_{m=0}^{\infty}\left(\frac{2}{\Gamma(2 m+1)} \int_{1}^{\infty}(\log \varpi)^{2 m} \mathbb{X}(\varpi) d \varpi\right)(s-6)^{2 m} \\
& =\sum_{m=0}^{\infty} \beta_{m}(s-6)^{2 m},
\end{aligned}
$$

where

$$
\beta_{m}=\frac{2}{\Gamma(2 m+1)} \int_{1}^{\infty}(\log \varpi)^{2 m} \mathbb{X}(\varpi) d \varpi .
$$

Thus, the result follows.

Theorem 3. Let $\Delta_{\tau}(\varpi)=\sum_{k=1}^{\infty} \tau(k) e^{k \varpi}$. If $\Xi_{\tau}(t)$ is defined in (9), then we have for $t \in \mathbb{R}$

$$
\Xi_{\tau}(t)=2 \int_{1}^{\infty} \cos (t \log \varpi) \mathbb{X}(\varpi) d \varpi .
$$

Proof. Making use of (9) and Theorem 1, we give

$$
\begin{aligned}
& \Xi_{\tau}(t)=\xi_{\tau}(6+i t) \\
& =2 \int_{1}^{\infty} \cosh \{[(6+i t)-6] \log \varpi\} \mathbb{X}(\varpi) d \varpi \\
& =2 \int_{1}^{\infty} \cosh (i t \log \varpi) \mathbb{X}(\varpi) d \varpi \\
& =2 \int_{1}^{\infty} \cos (t \log \varpi) \mathbb{X}(\varpi) d \varpi,
\end{aligned}
$$

and the result follows.

Remark. In 1929, Wilton (see [10], formulas (5.31) and (5.32)) proved that

$$
\Xi_{\tau}(t)=\int_{-\infty}^{\infty} \Lambda(l) e^{i l t} d l
$$


where

$$
\Lambda(l)=e^{6 l} G\left(e^{-2 \pi e^{l}}\right) .
$$

It has been proved by Ferguson and coauthors [18] that

$$
\Xi_{\tau}(t)=2 \int_{0}^{\infty} e^{6 x} \Delta_{\tau}\left(i e^{x}\right) \cos (t x) d x
$$

which can be rewritten as (27) when $x=\log \varpi$.

Thus, we find that

$$
\begin{aligned}
& \Xi_{\tau}(t) \\
& =\int_{-\infty}^{\infty} e^{6 l} G\left(e^{-2 \pi e^{l}}\right) e^{i l t} d l \\
& =2 \int_{0}^{\infty} e^{6 x} \Delta_{\tau}\left(i e^{x}\right) \cos (t x) d x \\
& =2 \int_{1}^{\infty} \cos (t \log \varpi) \mathbb{X}(\varpi) d \varpi .
\end{aligned}
$$

Theorem 4. Let $\Delta_{\tau}(\varpi)=\sum_{k=1}^{\infty} \tau(k) e^{k \varpi}$. If $\Xi_{\tau}(t)$ is defined in (9), then we have for $m \in \mathbb{N} \cup\{0\}$,

$$
\Xi_{\tau}(t)=\sum_{m=0}^{\infty}(-1)^{m} \beta_{m} t^{2 m}
$$

where

$$
\beta_{m}=\frac{2}{\Gamma(2 m+1)} \int_{1}^{\infty}(\log \varpi)^{2 m} \mathbb{X}(\varpi) d \varpi
$$

Proof. By Theorem 2 and (9), one obtains

$$
\Xi_{\tau}(t)=\xi_{\tau}(6+i t)=\sum_{m=0}^{\infty} \beta_{m}[(6+i t)-6]^{2 m}=\sum_{m=0}^{\infty}(-1)^{m} \beta_{m} t^{2 m}
$$

where

$$
\beta_{m}=\frac{2}{\Gamma(2 m+1)} \int_{1}^{\infty}(\log \varpi)^{2 m} \mathbb{X}(\varpi) d \varpi .
$$

Thus, we finish the proof. 
Remark. It is clearly seen that Theorem 3 also reduces to the desired result.

By (27) and (29), we find that

$$
\Xi_{\tau}(-t)=\Xi_{\tau}(t)
$$

which is in agreement with the result of Conrey and Ghosh [12].

Moreover, there exist

$$
\beta_{m}=\left[\frac{d^{2 m} \Xi_{\tau}(t)}{d t^{2 m}}\right]_{t=0}=\Xi_{\tau}^{(2 m)}(0)
$$

such that

$$
\Xi_{\tau}(t)=\sum_{m=0}^{\infty}(-1)^{m} \Xi_{\tau}^{(2 m)}(0) t^{2 m}
$$

In view of (34) and (30), we arrive at

$$
\beta_{m}=\left[\frac{d^{2 m} \Xi_{\tau}(t)}{d t^{2 m}}\right]_{t=0}=\Xi_{\tau}^{(2 m)}(0)=\frac{2}{\Gamma(2 m+1)} \int_{1}^{\infty}(\log \varpi)^{2 m} \mathbb{X}(\varpi) d \varpi .
$$

Similarly, we also present

$$
\beta_{m}=(-1)^{m}\left[\frac{d^{2 m} \xi_{\tau}(s)}{d s^{2 m}}\right]_{s=6}=(-1)^{m} \xi_{\tau}^{(2 m)}(6) .
$$

By using the above remark, one has the following corollaries:

Corollary 1. If $\Xi_{\tau}(t)$ is defined in (9), then we have for $m \in \mathbb{N} \cup\{0\}$,

$$
\Xi_{\tau}(t)=\sum_{m=0}^{\infty}(-1)^{m} \beta_{m} t^{2 m}
$$

where

$$
\beta_{m}=\Xi_{\tau}^{(2 m)}(0)
$$

Corollary 2. If $\xi_{\tau}(s)$ is defined in (4), then we have for $m \in \mathbb{N} \cup\{0\}$,

$$
\xi_{\tau}(s)=\sum_{m=0}^{\infty} \beta_{m}(s-6)^{2 m}
$$

where

$$
\beta_{m}=(-1)^{m} \xi_{\tau}^{(2 m)}(6) .
$$

In 1955 , Lekkerkerker [16] showed that $\Xi_{\tau}(t)$ has infinitely many zeros by Lekkerkerker theorem. 
Lemma 1. (Lekkerkerker theorem) If $\Xi_{\tau}(t)$ is defined in (9) and

$$
N_{0}(T)=\#\left\{s=6+i t: 0 \leq t \leq T, \xi_{\tau}(s)=0\right\}
$$

then we have

$$
N_{0}(T)>A T
$$

such that $\Xi_{\tau}(t)$ has infinitely many zeros, where $A$ is a positive constant.

Proof. See [16].

\section{The Product Formulas}

In this section we propose a variant for the Conrey-Ghosh product for the entire Ramanujan zeta-function and some product for Ramanujan zeta-function.

Now, we introduce the Conrey-Ghosh product by applying the result of the Weierstrass and Hadamard [11].

Lemma 2. (Conrey-Ghosh product)

Let $\rho_{\vartheta} \in \mathbb{C}, s \in \mathbb{C}$ and $\vartheta \in \mathrm{N}$. Suppose that $\rho_{\vartheta}$ run the nontrivial zeros of $\xi_{\tau}(s)$ and $\gamma$ is a constant. If $\xi_{\tau}(s)$ is defined in (4), then we have

$$
\xi_{\tau}(s)=\xi_{\tau}(0) e^{\gamma s} \prod_{\vartheta=1}^{\infty}\left(1-\frac{s}{\rho_{\vartheta}}\right) e^{s / \rho_{\vartheta}} .
$$

Proof. For the proof of the Conrey-Ghosh product, see [2].

Theorem 5. Let $\rho_{\vartheta} \in \mathbb{C}, s \in \mathbb{C}$ and $\vartheta \in \mathrm{N}$. Suppose that $\rho_{\vartheta}$ run the nontrivial zeros of $\xi_{\tau}(s)$ and $\gamma$ is a constant defined in (6). If $\xi_{\tau}(s)$ is defined in (4) and $\xi_{\tau}(0) \neq 0$, then we have

$$
\xi_{\tau}(6) \neq 0
$$

and

$$
\xi_{\tau}(12) \neq 0
$$

Proof. By (5) and Lemma 2, one has

$$
\xi_{\tau}(s)=\xi_{\tau}(12-s)
$$

such that

$$
\xi_{\tau}(12)=\xi_{\tau}(0) \neq 0
$$

and

$$
\xi_{\tau}(6)=\xi_{\tau}(0) e^{6 \gamma} \prod_{\vartheta=1}^{\infty}\left(1-\frac{6}{\rho_{\vartheta}}\right) e^{6 / \rho_{\vartheta}} \neq 0
$$


since $\rho_{\vartheta} \neq 6 \in \mathbb{R}$.

Thus, we complete the proof of Theorem 5.

Theorem 6. Let $\rho_{\vartheta} \in \mathbb{C}$ and $\vartheta \in \mathbb{N}$. Suppose that $\rho_{\vartheta}$ run the nontrivial zeros of $\xi_{\tau}(s)$ and $\gamma$ is a constant. If $\xi_{\tau}(s)$ is defined in (4) and $\Xi_{\tau}(t)$ is defined in (9), then we have

$$
\xi_{\tau}(s)=\xi_{\tau}(6) e^{\gamma(s-6)} \prod_{\vartheta=1}^{\infty}\left(1-\frac{s-6}{\rho_{\vartheta}-6}\right) e^{(s-6) / \rho_{\vartheta}}
$$

where $s \in \mathbb{C}$, and

$$
\Xi_{\tau}(t)=\xi_{\tau}(6) e^{i \gamma t} \prod_{\vartheta=1}^{\infty}\left(1-\frac{i t}{\rho_{\vartheta}-6}\right) e^{i t / \rho_{\vartheta}},
$$

where $t \in \mathbb{R}$.

Proof. With Lemma 2 and (48) one gives

$$
\begin{aligned}
& \xi_{\tau}(s) \\
& =\xi_{\tau}(0) e^{\gamma s} \prod_{\vartheta=1}^{\infty}\left(1-\frac{s}{\rho_{\vartheta}}\right) e^{s / \rho_{\vartheta}} \\
& =\xi_{\tau}(0) e^{\gamma s} \prod_{\vartheta=1}^{\infty} \frac{\rho_{\vartheta}-s}{\rho_{\vartheta}} e^{s / \rho_{\vartheta}} \\
& =\xi_{\tau}(0) e^{\gamma s} \prod_{\vartheta=1}^{\infty}\left(\frac{\rho_{\vartheta}-6}{\rho_{\vartheta}-6} \cdot \frac{\rho_{\vartheta}-s}{\rho_{\vartheta}}\right) e^{s / \rho_{\vartheta}} \\
& =\xi_{\tau}(0) e^{\gamma s} \prod_{\vartheta=1}^{\infty}\left(\frac{\rho_{\vartheta}-6}{\rho_{\vartheta}} \cdot \frac{\rho_{\vartheta}-s}{\rho_{\vartheta}-6}\right) e^{s / \rho_{\vartheta}} \\
& =\xi_{\tau}(0) e^{\gamma s} \prod_{\vartheta=1}^{\infty}\left(\frac{\rho_{\vartheta}-6}{\rho_{\vartheta}} \cdot \frac{\rho_{\vartheta}-s}{\rho_{\vartheta}-6}\right) e^{s / \rho_{\vartheta}} \\
& =\xi_{\tau}(0) e^{\gamma s} \prod_{\vartheta=1}^{\infty} \frac{\rho_{\vartheta}-6}{\rho_{\vartheta}} \prod_{\vartheta=1}^{\infty} \frac{\rho_{\vartheta}-s}{\rho_{\vartheta}-6} e^{s / \rho_{\vartheta}} \\
& =\xi_{\tau}(0) e^{\gamma s} \prod_{\vartheta=1}^{\infty}\left(1-\frac{6}{\rho_{\vartheta}}\right) \prod_{\vartheta=1}^{\infty} \frac{\rho_{\vartheta}-6-(s-6)}{\rho_{\vartheta}-6} e^{s / \rho_{\vartheta}} \\
& =\xi_{\tau}(0) e^{\gamma s} \prod_{\vartheta=1}^{\infty}\left(1-\frac{6}{\rho_{\vartheta}}\right) \prod_{\vartheta=1}^{\infty}\left(1-\frac{s-6}{\rho_{\vartheta}-6}\right) e^{s / \rho_{\vartheta}} \\
& =\xi_{\tau}(6) e^{\gamma(s-6)} \prod_{\vartheta=1}^{\infty}\left(1-\frac{s-6}{\rho_{\vartheta}-6}\right) e^{(s-6) / \rho_{\vartheta}},
\end{aligned}
$$

which completes the proof of (49).

Putting $s=6+i t$ into (49), one obtains (50).

Thus, we finish the proof of Theorem 6 . 
Theorem 7. Let $\rho_{\vartheta} \in \mathbb{C}$ and $\vartheta \in \mathbb{N}$. Suppose that $\rho_{\vartheta}$ run the nontrivial zeros of $\xi_{\tau}(s)$ and $\gamma$ is a constant. Then we have for $s \in \mathbb{C}$,

$$
L_{\tau}(s)=\frac{\xi_{\tau}(0)(2 \pi)^{s} e^{\gamma s}}{\Gamma(s)} \prod_{\vartheta=1}^{\infty}\left(1-\frac{s}{\rho_{\vartheta}}\right) e^{s / \rho_{\vartheta}},
$$

and

$$
L_{\tau}(s)=\frac{\xi_{\tau}(6)(2 \pi)^{s} e^{\gamma(s-6)}}{\Gamma(s)} \prod_{\vartheta=1}^{\infty}\left(1-\frac{s-6}{\rho_{\vartheta}-6}\right) e^{(s-6) / \rho_{\vartheta}} .
$$

Proof. By (4) and Lemma 2, one obtains

$$
L_{\tau}(s)=\frac{\xi_{\tau}(0)(2 \pi)^{s} e^{\gamma s}}{\Gamma(s)} \prod_{\vartheta=1}^{\infty}\left(1-\frac{s}{\rho_{\vartheta}}\right) e^{s / \rho_{\vartheta}},
$$

and by (4) and Theorem 6 , one gives

$$
\zeta_{\tau}(s)=\frac{\xi_{\tau}(6)(2 \pi)^{s} e^{\gamma(s-6)}}{\Gamma(s)} \prod_{\vartheta=1}^{\infty}\left(1-\frac{s-6}{\rho_{\vartheta}-6}\right) e^{(s-6) / \rho_{\vartheta}} .
$$

Thus, we finish the proof of Theorem $\%$.

Remark. By Theorem 7, it is clearly shown that $L_{\tau}(s)$ has the trivial zeros $s=-w$ with $w \in \mathbb{N} \cup\{0\}$, controlled by poles of $\Gamma(s)$, and that $\rho_{\vartheta}$ run the nontrivial zeros of $L_{\tau}(s)$ (for more details, see [3], p.174). Hardy proved in 1940 (see [3], p.174) that $L_{\tau}(11 / 2+i t) \neq 0$ and that $L_{\tau}(13 / 2+i t) \neq 0$ for $t \in \mathbb{R}$.

\section{Real zeros}

In this section we will prove that all of the zeros for the Ramanujan $\Xi$-function $\Xi_{\tau}(t)$ are real by the Lekkerkerker theorem and Theorem 6 .

Corollary 3. Let $t \in \mathbb{R}$. If $\Xi_{\tau}(t)$ is defined in (9), then all of the zeros of $\Xi_{\tau}(t)$ are real.

Proof. Applying Theorem 6, we have

$$
\Xi_{\tau}(t)=\Xi_{\tau}(0) e^{i \gamma t} \prod_{\vartheta=1}^{\infty}\left(1-\frac{i t}{\rho_{\vartheta}-6}\right) e^{i t / \rho_{\vartheta}},
$$

where $t \in \mathbb{R}, \vartheta \in \mathbb{N}$, and the product runs over zeroes $\rho_{\vartheta}$ of the entire Ramanujan zeta-function $\xi_{\tau}(s)$.

In order to investigate the zeros of $\Xi_{\tau}(t)$, we now rewrite (56) as

$$
\Xi_{\tau}(t)=\Xi_{\tau}(0) e^{i \gamma t} \prod_{\vartheta=1}^{\infty}\left(1-\frac{i t}{\psi_{\vartheta}}\right) e^{i t / \rho_{\vartheta}},
$$


where $t \in \mathbb{R}, \vartheta \in \mathbb{N}$, and the product runs over the zeroes $\psi_{\vartheta}$ of $\Xi_{\tau}(t)$ and the zeroes $\rho_{\vartheta}$ of the entire Ramanujan zeta-function $\xi_{\tau}(s)$.

Now, let us consider the first hypothesis that all zeros of $\Xi_{\tau}(t)$ are given by

$$
\psi_{\vartheta}=\widetilde{\sigma}_{\vartheta}+i \widetilde{\tau}_{\vartheta}
$$

where $\widetilde{\sigma}_{\vartheta} \in \mathbb{R} \backslash\{0\}$ and $\widetilde{\tau}_{\vartheta} \in \mathbb{R} \backslash\{0\}$, and the second hypothesis that all zeros of $\Xi_{\tau}(t)$ are

$$
\psi_{\vartheta}=\ell_{\vartheta}
$$

where $\ell_{\vartheta} \in \mathbb{R} \backslash\{0\}$. Our idea is that if two cases are false, then we have that all zeros of $\Xi_{\tau}(t)$ are real.

We now start to prove that they are false.

Hypothesis 1.

Now, we consider the first hypothesis that the complex zeros for $\Xi_{\tau}(t)$ can be expressed in the form

$$
\psi_{\vartheta}=\widetilde{\sigma}_{\vartheta}+i \widetilde{\tau}_{\vartheta}
$$

where $\widetilde{\sigma}_{\vartheta} \in \mathbb{R} \backslash\{0\}$ and $\widetilde{\tau}_{\vartheta} \in \mathbb{R} \backslash\{0\}$.

By (57) and (60), we now consider

$$
\begin{aligned}
& \Xi_{\tau}(t) \\
& =\Xi_{\tau}(0) e^{i \gamma t} \prod_{\vartheta=1}^{\infty}\left(1-\frac{i t}{\widetilde{\sigma}_{\vartheta}+i \widetilde{\tau}_{\vartheta}}\right) e^{i t / \rho_{\vartheta}} \\
& =\Xi_{\tau}(0) e^{i \gamma t} \prod_{\vartheta=1}^{\infty}\left(1-\frac{i t}{\psi_{\vartheta}}\right) e^{i t / \rho_{\vartheta}} .
\end{aligned}
$$

Putting $t=(s-6) / i$ into $(6)$, we now give

$$
\begin{aligned}
& \xi_{\tau}(s)=\Xi_{\tau}((s-6) / i) \\
& =\xi_{\tau}(6) e^{\gamma(s-6)} \prod_{\vartheta=1}^{\infty}\left(1-\frac{s-6}{\widetilde{\sigma}_{\vartheta}+i \widetilde{\tau}_{\vartheta}}\right) e^{(s-6) / \rho_{\vartheta}} \\
& =\xi_{\tau}(6) e^{\gamma(s-6)} \prod_{\vartheta=1}^{\infty}\left(1-\frac{s-6}{\psi_{\vartheta}}\right) e^{(s-6) / \rho_{\vartheta}}
\end{aligned}
$$

since $\Xi_{\tau}(0)=\xi_{\tau}(6)$.

Let $\rho_{\vartheta}=\operatorname{Re}\left(\rho_{\vartheta}\right)+i \psi_{\vartheta}$ run all roots of $\xi_{\tau}(s)=0$, where $\operatorname{Re}\left(\rho_{\vartheta}\right) \in \mathbb{R} \backslash\{0\}$ and $\psi_{\vartheta} \in \mathbb{R} \backslash\{0\}$. Then, we have from (62) that

$$
\xi_{\tau}\left(\rho_{\vartheta}\right)=\xi_{\tau}(6) e^{\gamma\left(\rho_{\vartheta}-6\right)} \prod_{n=1}^{\infty}\left(1-\frac{\rho_{\vartheta}-6}{\widetilde{\sigma}_{\vartheta}+i \widetilde{\tau}_{\vartheta}}\right) e^{\left(\rho_{\vartheta}-6\right) / \rho_{\vartheta}}=0
$$

where $\xi_{\tau}(6) \neq 0$.

From (63) we show

$$
\widetilde{\sigma}_{\vartheta}+i \widetilde{\tau}_{\vartheta}-\left(\rho_{\vartheta}-6\right)=0
$$


since $\xi_{\tau}(6) \neq 0$ and $e^{\gamma\left(\rho_{\vartheta}-6\right)} \prod_{n=1}^{\infty} e^{\left(\rho_{\vartheta}-6\right) / \rho_{\vartheta}} \neq 0$.

By (64), we present

$$
s_{\vartheta}=\operatorname{Re}\left(\rho_{\vartheta}\right)+i \psi_{\vartheta}=\widetilde{\sigma}_{\vartheta}+6+i \widetilde{\tau}_{\vartheta},
$$

where $\widetilde{\sigma}_{\vartheta} \in \mathbb{R} \backslash\{0\}$ and $\widetilde{\tau}_{\vartheta} \in \mathbb{R} \backslash\{0\}$.

By $\widetilde{\sigma}_{\vartheta} \in \mathbb{R} \backslash\{0\}$ and (65), that the critical line is expressed as

$$
\operatorname{Re}\left(s_{\vartheta}\right)=\tilde{\sigma}_{\vartheta}+6
$$

which is in contradiction with Lemma 1 (Lekkerkerker theorem), which states infinitely many zeros for $\xi_{\tau}(s)$ lie on the critical line $R e(s)=6$.

Hypothesis 2.

Now, we give the hypothesis that $\Xi_{\tau}(t)$ has zeros

$$
\psi_{\vartheta}=\ell_{\vartheta}
$$

where $\ell_{\vartheta} \in \mathbb{R} \backslash\{0\}$.

By inserting (67) into (57), we suggest that

$$
\Xi_{\tau}(t)=\Xi_{\tau}(0) e^{i \gamma t} \prod_{\vartheta=1}^{\infty}\left(1-\frac{i t}{\ell_{\vartheta}}\right) e^{i t / \rho_{\vartheta}} .
$$

Substituting $t=(s-6) / i$ and (68), we give

$$
\begin{aligned}
& \xi_{\tau}(s) \\
& =\Xi_{\tau}((s-1 / 2) / i) \\
& =\Xi_{\tau}(0) e^{i \gamma t} \prod_{\vartheta=1}^{\infty}\left[1-\frac{i[(s-6) / i]}{\ell_{\vartheta}}\right] e^{i t / \rho_{\vartheta}} \\
& =\xi_{\tau}(6) e^{\gamma(s-6)} \prod_{\vartheta=1}^{\infty}\left(1-\frac{s-6}{\ell_{\vartheta}}\right) e^{(s-6) / \rho_{\vartheta}}
\end{aligned}
$$

since $\Xi_{\tau}(0)=\xi_{\tau}(6)$.

Let $\rho_{\vartheta}=\operatorname{Re}\left(\rho_{\vartheta}\right)+i \psi_{\vartheta}$ such that $\xi_{\tau}\left(s_{\vartheta}\right)=0$, where $\operatorname{Re}\left(\rho_{\vartheta}\right) \in \mathbb{R} \backslash\{0\}$ and $\psi_{\vartheta} \in$ $\mathbb{R} \backslash\{0\}$.

From (69), we show that

$$
\xi_{\tau}\left(\rho_{\vartheta}\right)=\xi_{\tau}(6) e^{\gamma\left(\rho_{\vartheta}-6\right)} \prod_{\vartheta=1}^{\infty}\left(1-\frac{\rho_{\vartheta}-6}{\ell_{\vartheta}}\right) e^{\left(\rho_{\vartheta}-6\right) / \rho_{\vartheta}}=0 .
$$

From (70) we have that

$$
\rho_{\vartheta}-6-\ell_{\vartheta}=0
$$

Since $\ell_{\vartheta} \in \mathbb{R} \backslash\{0\},(71)$ implies

$$
\rho_{\vartheta}=6+\ell_{\vartheta} \in \mathbb{R},
$$


which is in contradiction with the well-known fact

$$
\rho_{\vartheta}=\operatorname{Re}\left(\rho_{\vartheta}\right)+i \psi_{\vartheta} \in \mathbb{C},
$$

where $\operatorname{Re}\left(\rho_{\vartheta}\right) \in \mathbb{R} \backslash\{0\}$ and $\psi_{\vartheta} \in \mathbb{R} \backslash\{0\}$.

Moreover, (72) and Lemma 1 (Lekkerkerker theorem) conflict with each other.

In sum, all zeros of $\Xi_{\tau}(t)$ are real since two cases are false.

Hence, we finish the proof.

Remark. We have to point out that Corollary 3 is a sufficient condition for (9) to have all infinitely many real zeros.

\section{THE NONTRIVIAL ZEROS}

In this section we present the detailed account of the proof of Conjecture 1.

To begin with, we consider the product of the Ramanujan $\Xi$-function with the aid of Corollary 3.

By Theorems 4 and 6 , we now structure the entire Ramanujan $\Xi$-function by

$$
\Xi_{\tau}(t)=\xi_{\tau}(6) e^{i \gamma t} \prod_{\vartheta=1}^{\infty}\left(1-\frac{i t}{\rho_{\vartheta}-6}\right) e^{i t / \rho_{\vartheta}}=\sum_{m=0}^{\infty}(-1)^{m} \beta_{m} t^{2 m},
$$

where $\beta_{m}$ are the coefficients and $\gamma$ is the constant.

In view of Corollary 3, the second term of (74) gives us to show that all of the zeros of $\Xi_{\tau}(t)$ are real.

By (75), we have $\psi_{\vartheta} \in \mathbb{C}$ such that

$$
\Xi_{\tau}\left(\psi_{\vartheta}\right)=0,
$$

which leads to

$$
\xi_{\tau}(6) e^{i \gamma \psi_{\vartheta}} \prod_{\vartheta=1}^{\infty}\left(1-\frac{i \psi_{\vartheta}}{\rho_{\vartheta}-6}\right) e^{i t / \rho_{\vartheta}}=0,
$$

or, alternatively,

$$
\sum_{m=0}^{\infty}(-1)^{m} \beta_{m}\left(\psi_{\vartheta}\right)^{2 m}=\sum_{m=0}^{\infty}(-1)^{m} \beta_{m} \psi_{\vartheta}^{2 m}=0 .
$$

With (76) and Theorem 5 we arrive at

$$
1-\frac{i \psi_{\vartheta}}{\rho_{\vartheta}-6}=0
$$

where $\xi_{\tau}(6) \neq 0$ and $\vartheta \in \mathbb{N}$.

From (78) one gets

$$
\rho_{\vartheta}=6+i \psi_{\vartheta}
$$


for $\vartheta \in \mathbb{N}$.

Substituting (79) into (50) and (53), we have

$$
\begin{aligned}
& \Xi_{\tau}(t) \\
& =\xi_{\tau}(6) e^{i \gamma t} \prod_{\vartheta=1}^{\infty}\left(1-\frac{i t}{\rho_{\vartheta}-6}\right) e^{i t / \rho_{\vartheta}} \\
& =\xi_{\tau}(6) e^{i \gamma t} \prod_{\vartheta=1}^{\infty}\left[1-\frac{i t}{\left(6+i \psi_{\vartheta}\right)-6}\right] e^{i t /\left(6+i \psi_{\vartheta}\right)} \\
& =\xi_{\tau}(6) e^{i \gamma t} \prod_{\vartheta=1}^{\infty}\left(1-\frac{i t}{i \psi_{\vartheta}}\right) e^{i t /\left(6+i \psi_{\vartheta}\right)} \\
& =\xi_{\tau}(6) e^{i \gamma t} \prod_{\vartheta=1}^{\infty}\left(1-\frac{t}{\psi_{\vartheta}}\right) e^{i t /\left(6+i \psi_{\vartheta}\right)}
\end{aligned}
$$

and

$$
\begin{aligned}
& \xi_{\tau}(s) \\
& =\xi_{\tau}(6) e^{\gamma(s-6)} \prod_{\vartheta=1}^{\infty}\left(1-\frac{s-6}{\rho_{\vartheta}-6}\right) e^{(s-6) / \rho_{\vartheta}} \\
& =\xi_{\tau}(6) e^{\gamma(s-6)} \prod_{\vartheta=1}^{\infty}\left[1-\frac{s-6}{\left(6+i \psi_{\vartheta}\right)-6}\right] e^{(s-6) /\left(6+i \psi_{\vartheta}\right)} \\
& =\xi_{\tau}(6) e^{\gamma(s-6)} \prod_{\vartheta=1}^{\infty}\left[1-\frac{s-6}{i \psi_{\vartheta}}\right] e^{(s-6) /\left(6+i \psi_{\vartheta}\right)}
\end{aligned}
$$

Similarly, by (79), (53) can be rewritten in the form

$$
\zeta_{\tau}(s)=\frac{\xi_{\tau}(6)(2 \pi)^{s} e^{\gamma(s-6)}}{\Gamma(s)} \prod_{\vartheta=1}^{\infty}\left[1-\frac{s-6}{i \psi_{\vartheta}}\right] e^{(s-6) /\left(6+i \psi_{\vartheta}\right)}
$$

From (81) and (82), one also has (78) and (79) once again.

Thus, we finish the proof of Conjecture 1.

\section{The EQUivalent THEOREMS}

In this section we propose some equivalent theorems for the Ramanujan zetafunction based on the true of Conjecture 1.

From (79) we find that $\rho_{\vartheta}=6+i \psi_{\vartheta}, \overline{\rho_{\vartheta}}=6-i \psi_{\vartheta}, 12-\rho_{\vartheta}=6-i \psi_{\vartheta}$, and $1-\overline{\rho_{\vartheta}}=6+i \psi_{\vartheta}$ are the zeros for the entire Ramanujan zeta-function and the nontrivial zeros for the Ramanujan zeta-function. 
By $(80)$ and $\varphi_{\vartheta}=\left|\psi_{\vartheta}\right|$, we have

$$
\begin{aligned}
& \Xi_{\tau}(t) \\
& =\xi_{\tau}(6) e^{i \gamma t} \prod_{\vartheta=1}^{\infty}\left(1-\frac{t}{\psi_{\vartheta}}\right) e^{i t /\left(6+i \psi_{\vartheta}\right)} \\
& =\xi_{\tau}(6) e^{i \gamma t} \prod_{\vartheta=1}^{\infty}\left(1-\frac{t}{\varphi_{\vartheta}}\right) e^{i t /\left(6+i \varphi_{\vartheta}\right)} \prod_{\vartheta=1}^{\infty}\left(1+\frac{t}{\varphi_{\vartheta}}\right) e^{i t /\left(6-i \varphi_{\vartheta}\right)} \\
& =\xi_{\tau}(6) e^{i \gamma t} \prod_{\vartheta=1}^{\infty}\left[\left(1-\frac{t}{\varphi_{\vartheta}}\right)\left(1+\frac{t}{\varphi_{\vartheta}}\right)\right] e^{i 12 t /\left(6+i \varphi_{\vartheta}\right)\left(6-i \varphi_{\vartheta}\right)} \\
& =\xi_{\tau}(6) e^{i \gamma t} \prod_{\vartheta=1}^{\infty}\left(1-\frac{t^{2}}{\varphi_{\vartheta}^{2}}\right) e^{i 12 t /\left(36+\varphi_{\vartheta}^{2}\right)}
\end{aligned}
$$

Because (83) is valid for $t \in \mathbb{R}$ and $\Xi_{\tau}(t)$ is the even function, one has

$$
\Xi_{\tau}(-t)=\xi_{\tau}(6) e^{-i \gamma t} \prod_{\vartheta=1}^{\infty}\left(1-\frac{t^{2}}{\varphi_{\vartheta}^{2}}\right) e^{-i 12 t /\left(36+\varphi_{\vartheta}^{2}\right)}
$$

and

$$
\Xi_{\tau}(t)=\xi_{\tau}(6) e^{i \gamma t} \prod_{\vartheta=1}^{\infty}\left(1-\frac{t^{2}}{\varphi_{\vartheta}^{2}}\right) e^{i 12 t /\left(36+\varphi_{\vartheta}^{2}\right)}
$$

such that

$$
\xi_{\tau}(6) e^{-i \gamma t} \prod_{\vartheta=1}^{\infty}\left(1-\frac{t^{2}}{\varphi_{\vartheta}^{2}}\right) e^{-i 12 t /\left(36+\varphi_{\vartheta}^{2}\right)}=\xi_{\tau}(6) e^{i \gamma t} \prod_{\vartheta=1}^{\infty}\left(1-\frac{t^{2}}{\varphi_{\vartheta}^{2}}\right) e^{i 12 t /\left(36+\varphi_{\vartheta}^{2}\right)} .
$$

Thus, by (86), one gets

$$
e^{2 i \gamma t} \prod_{\vartheta=1}^{\infty} e^{i 24 t /\left(36+\varphi_{\vartheta}^{2}\right)}=1
$$

which leads to

$$
2 i \gamma t+i 24 t \sum_{\vartheta=1}^{\infty} \frac{1}{36+\varphi_{\vartheta}^{2}}=0
$$

Thus,

$$
\sum_{\vartheta=1}^{\infty} \frac{1}{36+\varphi_{\vartheta}^{2}}=-\frac{\gamma}{12}
$$

and

$$
\Xi_{\tau}(t)=\xi_{\tau}(6) \prod_{\vartheta=1}^{\infty}\left(1-\frac{t^{2}}{\varphi_{\vartheta}^{2}}\right) .
$$


Moreover, (85) reduces to

$$
\Xi_{\tau}(0)=\xi_{\tau}(6) \neq 0 .
$$

Corollary 4. Suppose that $\Xi_{\tau}(t)$ is defined in (9), where $t \in \mathbb{R}$. Let $\vartheta \in \mathbb{N}$ such that $\psi_{\vartheta}$ run the zeros for $\Xi_{\tau}(t)$ and $\varphi_{\vartheta}=\left|\psi_{\vartheta}\right|$. Then we have

$$
\Xi_{\tau}(t)=\Xi_{\tau}(0) \prod_{\vartheta=1}^{\infty}\left(1-\frac{t^{2}}{\varphi_{\vartheta}^{2}}\right)
$$

and

$$
\Xi_{\tau}(t)=\Xi_{\tau}(0) \prod_{\vartheta=1}^{\infty}\left(1-\frac{t}{\psi_{\vartheta}}\right) .
$$

Proof. Combining (90) and (85), we carry out (92).

In view of (92), we have

$$
\begin{aligned}
& \Xi_{\tau}(t) \\
& =\Xi_{\tau}(0) \prod_{\vartheta=1}^{\infty}\left(1-\frac{t^{2}}{\varphi_{\vartheta}^{2}}\right) \\
& =\Xi_{\tau}(0) \prod_{\vartheta=1}^{\infty}\left[\left(1+\frac{t}{\varphi_{\vartheta}}\right)\left(1-\frac{t}{\varphi_{\vartheta}}\right)\right] \\
& =\Xi_{\tau}(0) \prod_{\vartheta=1}^{\infty}\left(1-\frac{t}{\psi_{\vartheta}}\right) .
\end{aligned}
$$

Thus, we finish the proof of Corollary 4.

Theorem 8. Let $\vartheta \in \mathbb{N}$ and $t \in \mathbb{R}$. The following representations for $\Xi_{\tau}(t)$ are equivalent:

(1A):

$$
\Xi_{\tau}(t)=\int_{0}^{\infty} \varpi^{5+i t} \Delta_{\tau}(i \varpi) d \varpi,
$$

where $\Delta_{\tau}(\varpi)$ is defined in (11);

(2A):

$$
\Xi_{\tau}(t)=2 \int_{1}^{\infty} \cos (t) \varpi^{5} \Delta_{\tau}(i \varpi) d \varpi
$$

where $\Delta_{\tau}(\varpi)$ is defined in (11); 
(3A):

$$
\Xi_{\tau}(t)=\sum_{m=0}^{\infty}(-1)^{m} \beta_{m} t^{2 m}
$$

where $\beta_{m}$ is defined in (36);

(4A):

$$
\Xi_{\tau}(t)=\xi_{\tau}(6) e^{i \gamma t} \prod_{\vartheta=1}^{\infty}\left(1-\frac{t}{\psi_{\vartheta}}\right) e^{i t /\left(6+i \psi_{\vartheta}\right)},
$$

where $\psi_{\vartheta}$ run the zeros for $\Xi_{\tau}(t)$ and $\gamma$ is defined in (89);

(5A):

$$
\Xi_{\tau}(t)=\Xi_{\tau}(0) \prod_{\vartheta=1}^{\infty}\left(1-\frac{t^{2}}{\varphi_{\vartheta}^{2}}\right)
$$

where $\varphi_{\vartheta}=\left|\psi_{\vartheta}\right|$;

(6A):

$$
\Xi_{\tau}(t)=\Xi_{\tau}(0) \prod_{\vartheta=1}^{\infty}\left(1-\frac{t}{\psi_{\vartheta}}\right),
$$

where $\psi_{\vartheta}$ run the zeros for $\Xi_{\tau}(t)$.

Proof. Eq. (95) (see (9) in Section 1) was proved by Conrey and Ghosh [12]. (96) is shown in Theorem 3, and (97) was presented in Theorem 4. (98) was given by Theorem 6 and Conjecture 1. (99) and (100) were presented in Corollary 4. Thus, we finish the proof of Theorem 8. These are what we wanted to show.

Theorem 9. Let $\vartheta \in \mathbb{N}$ and $s \in \mathbb{C}$. The following representations for $\xi_{\tau}(s)$ are equivalent:

(1B):

$$
\xi_{\tau}(s)=\int_{0}^{\infty} \varpi^{s-1} \Delta_{\tau}(i \varpi) d \varpi,
$$

where $\Delta_{\tau}(\varpi)$ is defined in (11);

(2B):

$$
\xi_{\tau}(s)=2 \int_{1}^{\infty} \cosh (t) \mathbb{X}(\varpi) d \varpi,
$$

where $\Delta_{\tau}(\varpi)$ is defined in (11); 
(3B):

$$
\xi_{\tau}(s)=\sum_{m=0}^{\infty} \beta_{m} t^{2 m}
$$

where $\beta_{m}$ is defined in (36);

(4B):

$$
\xi_{\tau}(s)=\xi_{\tau}(6) e^{\gamma(s-6)} \prod_{\vartheta=1}^{\infty}\left(1-\frac{s-6}{i \psi_{\vartheta}}\right) e^{(s-6) /\left(6+i \psi_{\vartheta}\right)},
$$

where $\psi_{\vartheta}$ run the zeros for $\Xi_{\tau}(t)$ and $\gamma$ is defined in (89);

(5B):

$$
\xi_{\tau}(s)=\xi_{\tau}(6) \prod_{\vartheta=1}^{\infty}\left(1+\frac{(s-6)^{2}}{\varphi_{\vartheta}^{2}}\right),
$$

where $\varphi_{\vartheta}=\left|\psi_{\vartheta}\right|$;

(6B):

$$
\xi_{\tau}(s)=\xi_{\tau}(6) \prod_{\vartheta=1}^{\infty}\left(1-\frac{s-6}{i \psi_{\vartheta}}\right)
$$

where $\psi_{\vartheta}$ run the zeros for $\Xi_{\tau}(t)$.

Proof. Eq. (101) (see (8) in Section 1) was proved in [12]. (102) was presented in Theorem 1, and (103) was presented in Theorem 2. (104) was proved by Theorem 6 and Conjecture 1. Putting $t=-i(s-6)$ into (99) and (100), one obtains

$$
\xi_{\tau}(s)=\Xi_{\tau}(0) \prod_{\vartheta=1}^{\infty}\left(1+\frac{(s-6)^{2}}{\varphi_{\vartheta}^{2}}\right)=\xi_{\tau}(6) \prod_{\vartheta=1}^{\infty}\left(1+\frac{(s-6)^{2}}{\varphi_{\vartheta}^{2}}\right),
$$

and

$$
\xi_{\tau}(s)=\Xi_{\tau}(0) \prod_{\vartheta=1}^{\infty}\left(1-\frac{s-6}{i \psi_{\vartheta}}\right)=\xi_{\tau}(6) \prod_{\vartheta=1}^{\infty}\left(1-\frac{s-6}{i \psi_{\vartheta}}\right),
$$

since (91) is valid.

Hence we complete of the proof.

Theorem 10. Let $\vartheta \in \mathbb{N}$ and $s \in \mathbb{C}$. The following representations for $L_{\tau}(s)$ are equivalent: 
(1C):

$$
L_{\tau}(s)=\frac{(2 \pi)^{s}}{\Gamma(s)} \int_{0}^{\infty} \varpi^{s-1} \Delta_{\tau}(i \varpi) d \varpi,
$$

where $\Delta_{\tau}(\varpi)$ is defined in (11);

$(2 \mathrm{C})$ :

$$
L_{\tau}(s)=\frac{2^{1+s} \pi^{s}}{\Gamma(s)} \int_{1}^{\infty} \cosh (t) \mathbb{X}(\varpi) d \varpi,
$$

where $\Delta_{\tau}(\varpi)$ is defined in (11);

(3C):

$$
L_{\tau}(s)=\frac{(2 \pi)^{s}}{\Gamma(s)} \sum_{m=0}^{\infty} \beta_{m} t^{2 m}
$$

where $\beta_{m}$ is defined in (36);

$(4 \mathrm{C})$ :

$$
L_{\tau}(s)=\frac{\xi_{\tau}(6)(2 \pi)^{s}}{\Gamma(s)} e^{\gamma(s-6)} \prod_{\vartheta=1}^{\infty}\left(1-\frac{s-6}{i \psi_{\vartheta}}\right) e^{(s-6) /\left(6+i \psi_{\vartheta}\right)},
$$

where $\psi_{\vartheta}$ run the zeros for $\Xi_{\tau}(t)$ and $\gamma$ is defined in (89);

$(5 \mathrm{C})$ :

$$
L_{\tau}(s)=\frac{\xi_{\tau}(6)(2 \pi)^{s}}{\Gamma(s)} \prod_{\vartheta=1}^{\infty}\left(1+\frac{(s-6)^{2}}{\varphi_{\vartheta}^{2}}\right),
$$

where $\varphi_{\vartheta}=\left|\psi_{\vartheta}\right|$;

$(6 \mathrm{C})$ :

$$
L_{\tau}(s)=\frac{\xi_{\tau}(6)(2 \pi)^{s}}{\Gamma(s)} \prod_{\vartheta=1}^{\infty}\left(1-\frac{s-6}{i \psi_{\vartheta}}\right),
$$

where $\psi_{\vartheta}$ run the zeros for $\Xi_{\tau}(t)$.

Proof. By (4), we have

$$
L_{\tau}(s)=\frac{(2 \pi)^{s}}{\Gamma(s)} \xi_{\tau}(s)
$$

and by (115) and Theorem 9, we obtain the required results.

Thus, the proof of Theorem 10 is finished. 
We now define

$$
\mathbb{M}_{\tau}(t)=L_{\tau}(6+i t)=\frac{(2 \pi)^{6+i t}}{\Gamma(6+i t)} \xi_{\tau}(6+i t)=\frac{(2 \pi)^{6+i t}}{\Gamma(6+i t)} \Xi_{\tau}(t)
$$

where $(2 \pi)^{6+i t} / \Gamma(6+i t) \neq 0$ for $t \in \mathbb{R}$.

Theorem 11. Let $\vartheta \in \mathbb{N}$ and $t \in \mathbb{R}$. The following representations for $\mathbb{M}_{\tau}(t)$ are equivalent:

(1D):

$$
\mathrm{M}_{\tau}(t)=\frac{(2 \pi)^{6+i t}}{\Gamma(6+i t)} \int_{0}^{\infty} \varpi^{5+i t} \Delta_{\tau}(i \varpi) d \varpi
$$

where $\Delta_{\tau}(\varpi)$ is defined in (11);

(2D):

$$
\mathbb{M}_{\tau}(t)=\frac{2^{7+i t} \pi^{6+i t}}{\Gamma(6+i t)} \int_{1}^{\infty} \cos (t) \mathbb{X}(\varpi) d \varpi,
$$

where $\Delta_{\tau}(\varpi)$ is defined in (11);

(3D):

$$
\mathrm{M}_{\tau}(t)=\frac{(2 \pi)^{6+i t}}{\Gamma(6+i t)} \sum_{m=0}^{\infty}(-1)^{m} \beta_{m} t^{2 m}
$$

(4D):

$$
\mathbb{M}_{\tau}(t)=\frac{(2 \pi)^{6+i t} \xi_{\tau}(6) e^{i \gamma t}}{\Gamma(6+i t)} \prod_{\vartheta=1}^{\infty}\left(1-\frac{t}{\psi_{\vartheta}}\right) e^{i t /\left(6+i \psi_{\vartheta}\right)},
$$

where $\psi_{\vartheta}$ run the zeros for $\Xi_{\tau}(t)$ and $\gamma$ is defined in (89);

(5D):

$$
\mathrm{M}_{\tau}(t)=\frac{\Xi_{\tau}(0)(2 \pi)^{6+i t}}{\Gamma(6+i t)} \prod_{\vartheta=1}^{\infty}\left(1-\frac{t^{2}}{\varphi_{\vartheta}^{2}}\right)
$$

where $\varphi_{\vartheta}=\left|\psi_{\vartheta}\right|$;

(6D):

$$
\mathrm{M}_{\tau}(t)=\frac{\Xi_{\tau}(0)(2 \pi)^{6+i t}}{\Gamma(6+i t)} \prod_{\vartheta=1}^{\infty}\left(1-\frac{t}{\psi_{\vartheta}}\right)
$$

where $\psi_{\vartheta}$ run the zeros for $\Xi_{\tau}(t)$. 
Proof. Combining (116) and Theorem 10, we arrive at the desired results.

Now, we consider that the Hafner theorem was conjectured by Hafner in 1983, as a statement equivalent to Conjecture 1 [13].

Theorem 12. (Hafner theorem)

Let

$$
N_{0}(T)=\#\left\{s=6+i t: 0 \leq t \leq T, \xi_{\tau}(s)=0\right\}
$$

and

$$
N(T)=\#\left\{s=\sigma+i t: 0 \leq t \leq T, 11 / 2<\sigma<13 / 2, \xi_{\tau}(s)=0\right\} .
$$

Then we have

$$
N_{0}(T)=N(T) .
$$

Proof. Let $0 \leq t \leq T$ and $11 / 2<\sigma<13 / 2$. With the aid of (79), one has

$$
\rho_{\vartheta}=6+i \varphi_{\vartheta}
$$

such that

$$
\Xi_{\tau}\left(\rho_{\vartheta}\right)=0
$$

Thus, the result follows.

Remark. By the above results, we show the following comments.

- By theory of the entire functions [20], it is observed that $\Xi_{\tau}(t)$ is an even entire function of order $\alpha=1$ with the exponent of convergence $\lambda=1$ and genus $\beta=0$, and of growth $(1,0) . \xi_{\tau}(s)$ is an entire function of order $\alpha=1$ with the exponent of convergence $\lambda=1$ and genus $\beta=0$, and is of growth $(1,0)$.

- It was proposed in 1940 by Hardy [3] that the nontrivial zeros $\rho_{\vartheta}$ for $L_{\tau}(s)$ lie on the critical line $\operatorname{Re}(s)=6$ and in the critical strip $11 / 2<\operatorname{Re}(s)<13 / 2$, and that the trivial zeros for $L_{\tau}(s)$ are $s=-w$ with $w \in \mathbb{N} \cup\{0\}$. The trivial and nontrivial zeros, critical line and critical trip for the Ramanujan zeta-function in the entire complex plane are shown in Fig. 1. Thus, it is clear to see that Conjecture 1 is true.

Acknowledgements. This work is supported by the Yue-Qi Scholar of the China University of Mining and Technology (No. 102504180004). 


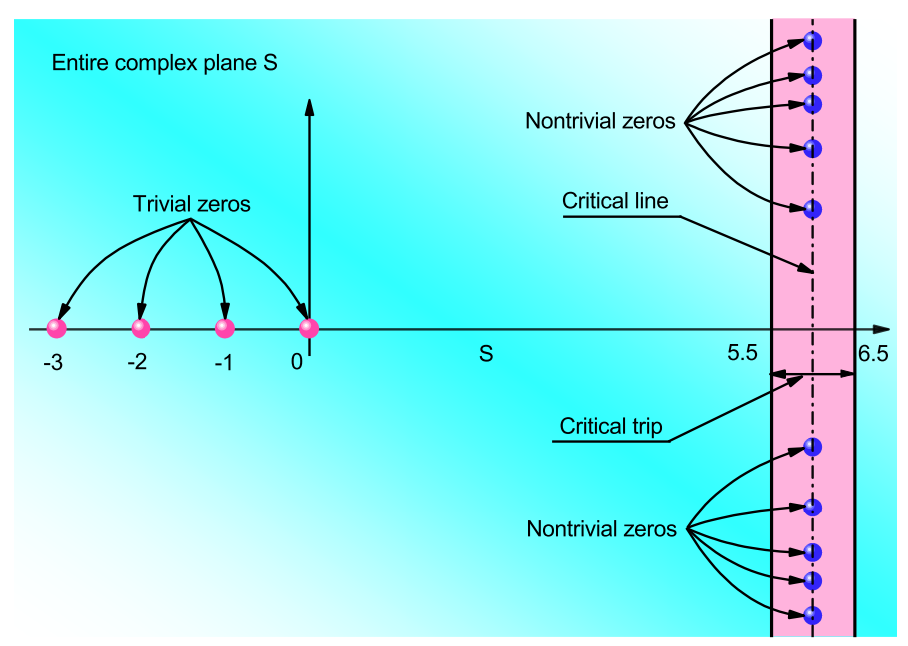

FiguRE 1. The blue points are represented as the nontrivial zeros for $L_{\tau}(s)$. The amaranth points are represented as the trivial zeros for $L_{\tau}(s)$. All nontrivial zeros for $L_{\tau}(s)$ lie on the critical line $R e(s)=6$ and in the critical strip $11 / 2<\operatorname{Re}(s)<13 / 2$.

\section{REFERENCES}

[1] Ramanujan, S. (1916). On certain arithmetical functions. Transactions of the Cambridge Philosophical Society, 22(9), 159-184.

[2] Conrey, J. B., Ghosh, A. (1988). Simple zeros of the Ramanujan $\tau$-Dirichlet series. Inventiones mathematicae, 94(2), 403-419.

[3] Hardy, G. H. (1940). Ramanujan twelve lectures on subjects suggested by his life and work. Cambridge University Press, Cambridge.

[4] Mordell, L. J. (1917). On Mr Ramanujan's empirical expansions of modular functions. Proceedings of the Cambridge Philosophical Society, 19, 117-124.

[5] Hardy, G. H. (1927). Note on Ramanujan's arithmetical function $\tau(n)$. Mathematical Proceedings of the Cambridge Philosophical Society, 23(06), 675-680.

[6] Deligne, P. (1974). La conjecture de Weil. I. Publications Mathématiques de l'Institut des Hautes Études Scientifiques, 43(1), 273-307.

[7] Moreno, C. J. (1974). A necessary and sufficient condition for the Riemann hypothesis for Ramanujan's zeta function. Illinois Journal of Mathematics, 18(1), 107-114.

[8] Fomenko, O. M. (1980). Applications of the theory of modular forms to number theory. Journal of Soviet Mathematics, 14(4), 1307-1362.

[9] Rankin, R. A. (1939). Contributions to the theory of Ramanujan's function $\tau(n)$ and similar arithmetical functions: I. The zeros of the function on the line. Mathematical Proceedings of the Cambridge Philosophical Society, 35(3), 351-356.

[10] Wilton, J. R. (1929). A note on Ramanujan's arithmetical function $\tau(n)$. Mathematical Proceedings of the Cambridge Philosophical Society, 25(2), 121-129.

[11] De Bruijn, N. G. (1950). The roots of trigonometric integrals. Duke Mathematical Journal, 17(3), 197-226. 
[12] Conrey, J. B., Ghosh, A. (1994). Turán inequalities and zeros of Dirichlet series associated with certain cusp forms. Transactions of the American Mathematical Society, 342(1), 407-419.

[13] Hafner, J. L. (1983). Zeros on the critical line for Dirichlet series attached to certain cusp forms. Mathematische Annalen, 264, 21-37.

[14] Köhler, G. (2011). Eta products and theta series identities. Springer, New York.

[15] Chirre, A., Castañón, O.V. (2020). A note on the zeros of approximations of the Ramanujan $\Xi$-function. The Ramanujan Journal, DOI: 10.1007/s11139-020-00321-7.

[16] Lekkerkerker, C. G. (1955). On the zeros of a class of Dirichlet series. van Gorcum.

[17] Ki, H. (2008). On the zeros of approximations of the Ramanujan $\Xi$-function. The Ramanujan Journal, 17(1), 123-143.

[18] Ferguson, H. R. P., Major, R. D., Powell, K. E., Throolin, H. G. (1984). On zeros of mellin transforms of $S L 2(\mathbf{Z})$ cusp forms. Mathematics of Computation, 42(165), 241-255.

[19] Keiper, J. B. (1996). On the zeros of the Ramanujan $\tau$-Dirichlet series in the critical strip. Mathematics of Computation, 65(216), 1613-1620.

[20] Boas, R. P. (1954). Entire functions. Academic Press, New York.

E-mail address: dyangxiaojun@163.com; xjyang@cumt.edu.cn

School of Mathematics, China University of Mining and Technology, Xuzhou 221116, CHINA 